\title{
Esophageal squamous cell carcinoma patients with positive lymph nodes benefit from extended radical lymphadenectomy
}

\author{
Bin Li, MD, ${ }^{\text {a,b }}$ Hong Hu, MD, ${ }^{\text {a,b }}$ Yawei Zhang, MD, ${ }^{\text {a,b }}$ Jie Zhang, MD, ${ }^{a, b}$ Yihua Sun, MD,, a, \\ Jiaqing Xiang, MD, a,b and Haiquan Chen, MD ${ }^{a, b}$
}

\begin{abstract}
Background: The usefulness of lymphadenectomy for advanced esophageal cancer is unclear. A post-hoc subgroup analysis involving a randomized controlled trial was performed to compare the long-term survival of patients with esophageal cancer and lymph nodes metastasis (LNM) after extended lymphadenectomy via the right thoracic approach with that of those undergoing limited lymphadenectomy via the left thoracic approach.
\end{abstract}

Methods: Between May 2010 and July 2012, 300 patients with thoracic esophageal cancer were randomized to undergo esophagectomy through either the right or left thoracic approach. Of these patients, 129 with esophageal squamous cell carcinoma and LNM by postoperative pathology were included in this analysis. Disease-free survival and overall survival (OS) were compared between the extended $(n=64)$ and limited $(n=65)$ lymphadenectomy groups after 5 years' follow-up. Cox regression analysis was used to identify the factors associated with OS.

Results: The 5-year disease-free survival rates were $40 \%$ and $26 \%$ in the extended and limited lymphadenectomy groups, respectively (hazard ratio [HR], 1.542; 95\% confidence interval [CI], 1.018-2.335; $P=.037)$, and the 5 -year OS rates were $51 \%$ and $31 \%$, respectively (HR, 1.719; 95\% CI, 1.111 $2.660 ; P=.013)$. The extended lymphadenectomy group had less locoregional recurrence $(\mathrm{HR}, 0.583 ; 95 \% \mathrm{CI}, 0.375-0.908 ; P=.015)$ and delayed distant metastasis (HR, 1.407; 95\% CI, 0.914-2.167; $P=.115$ ). Reduced OS was associated with limited lymphadenectomy, R1/2 resection margins, TNM stage IV, and lack of postoperative adjuvant therapy, as per the Cox regression analysis.

Conclusions: Improved long-term survival was observed in patients with esophageal squamous cell carcinoma and LNM after extended lymphadenectomy via the right thoracic approach. (J Thorac Cardiovasc Surg 2019;157:1275-83)

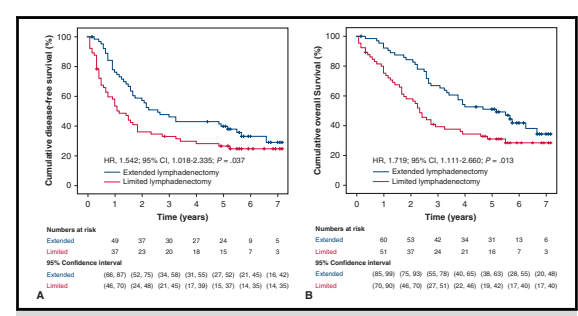

Extended lymphadenectomy improved cumulative probability of disease-free survival (A) and overall survival (B) in patients with esophageal squamous cell carcinoma and lymph nodes metastasis.

\section{Central Message}

Better OS and DFS were observed in patients with esophageal squamous cell carcinoma and lymph nodes metastasis after radical extended lymphadenectomy.

\section{Perspective}

This study compared extended and limited lymphadenectomy in patients with esophageal squamous cell carcinoma and positive lymph nodes. The results indicated the importance of radical lymphadenectomy in the treatment of esophageal cancer. Esophageal squamous cell carcinoma patients with positive lymph nodes benefit from extended radical lymphadenectomy.

See Commentaries on pages 1284 and 1286.
Esophageal cancer is notorious for early lymph nodes metastasis (LNM). Survival is greatly reduced when LNM occurs. ${ }^{1}$ The treatment strategy for esophageal cancer with LNM varies among countries. In Western countries, adenocarcinoma is the major histologic type, and

From the ${ }^{\mathrm{a} D e p a r t m e n t}$ of Thoracic Surgery, Fudan University Shanghai Cancer Center, Shanghai, China; and ${ }^{b}$ Department of Oncology, Shanghai Medical College, Fudan University, Shanghai, China.

Received for publication June 3, 2018; revisions received Oct 26, 2018; accepted for publication Nov 10, 2018.

Address for reprints: Haiquan Chen, MD, 270 Dong'an Rd, Shanghai 20032, China (E-mail: hqchen1@yahoo.com).

$0022-5223 / \$ 36.00$

Copyright (c) 2018 by The American Association for Thoracic Surgery

https://doi.org/10.1016/j.jtcvs.2018.11.094 neoadjuvant therapy has become standard treatment for the disease. ${ }^{2,3}$ In China, squamous cell carcinoma is the most common subtype, ${ }^{4}$ and transthoracic esophagectomy combined with adjuvant therapy is the main treatment strategy. However, during esophageal surgeries, the extent of lymphadenectomy varies around the world, and the role

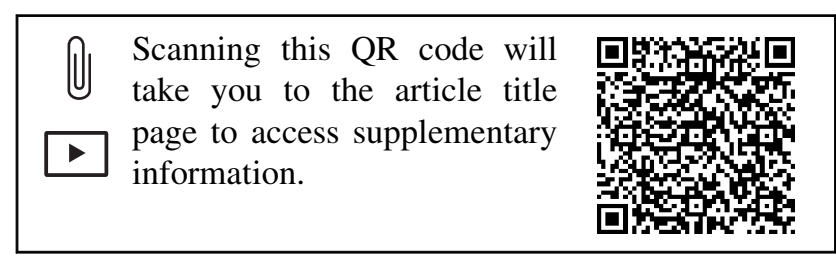




$$
\begin{aligned}
\text { Abbreviations and Acronyms } \\
\text { CI }=\text { confidence interval } \\
\text { CT }=\text { computed tomography } \\
\text { DFS }=\text { disease-free survival } \\
\text { HR }=\text { hazard ratio } \\
\text { IQR }=\text { interquartile range } \\
\text { LNM }=\text { lymph nodes metastasis } \\
\text { OS }=\text { overall survival } \\
\text { TNM }=\text { tumor-node-metastasis }
\end{aligned}
$$

of lymphadenectomy for patients with advanced esophageal cancer is still unclear.

In 2010, we initiated a randomized trial to compare extended radical lymphadenectomy through the right thoracic approach and limited lymphadenectomy through the left thoracic approach. ${ }^{5}$ The trial was designed to evaluate 3-year survival, and the results showed significant survival benefit from extended radical lymphadenectomy, particularly for those with LNM. $^{6}$ Currently, the last enrolled case has been followed for more than 5 years. Given the controversies on the treatment for advanced esophageal cancers, a post-hoc subgroup analysis of a randomized controlled trial for patients with positive lymph nodes was performed with updated follow-up periods to verify the impact of extended lymphadenectomy on cancers with LNM.

\section{PATIENTS AND METHODS \\ Patients}

The study protocol was approved by the institutional review board at $\mathrm{Fu}$ dan University Shanghai Cancer Center. This single-center, open-label, randomized trial was conducted at Fudan University Shanghai Cancer Center in China. Surgeries were performed by, or under the supervision of, 3 senior surgeons in our center (H.C., J.X., Y.Z.). All enrolled patients provided written informed consent. The study is registered at ClinicalTrials. gov (NCT01047111).

Eligibility criteria included resectable esophageal cancer (cT1-3, N0-1, M0 according to the sixth tumor-node-metastasis [TNM] classification system) in the middle or lower third of the thoracic esophagus (inferior to the carina and $3 \mathrm{~cm}$ superior to the cardia) and no evidence of distant metastases (including the absence of histologically confirmed tumor-positive cervical lymph nodes and unresectable celiac lymph nodes). The exclusion criteria included age $>75$ years, the presence of enlarged lymph nodes in the upper mediastinum ( $>5 \mathrm{~mm}$ ), history of other malignant diseases, previous gastric or esophageal surgery, neoadjuvant chemotherapy or radiotherapy, severe major organ dysfunction, and a Karnofsky index of $<80$.

The workup for assessing patient operability included a panel of oncologic evaluations (eg, upper gastrointestinal endoscopy with histologic examination, upper gastrointestinal barium swallow, computed tomography [CT] of the chest and upper abdomen, and ultrasound of the cervical region), and standard pulmonary and cardiac functional tests.

\section{Randomization}

The patients were assigned using simple randomization (1:1 ratio) with a computer-generated sequence to undergo either the right thoracic or left thoracic procedure. Concealment of group assignment was conducted

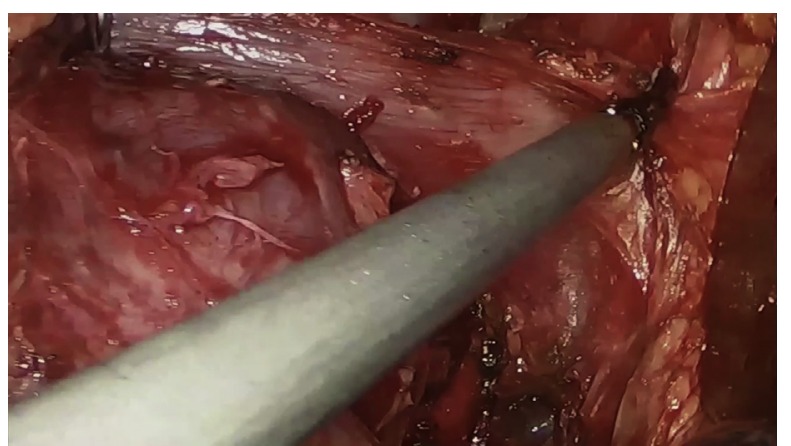

VIDEO 1. Lymph nodes dissection along the left recurrent nerve via right thoracic approach. Video available at: https://www.jtcvs.org/article/S00225223(18)33240-9/fulltext.

using opaque sealed envelopes. The envelopes were opened on the morning of the planned resection. The patients, surgeons, and assessors were aware of the assigned treatment. In this study, patients with squamous cell carcinoma and LNM, confirmed using postoperative pathological examination, were included for this post-hoc subgroup analysis.

\section{Right Thoracic Procedure}

The patient was initially placed in the supine position. Through an upper midline abdominal incision, gastric tubulization was performed and a feeding jejunostomy was inserted. During the abdominal phase, the lymph nodes in the perigastric region were removed, including the nodes along the left gastric artery, splenic artery, common hepatic artery, and celiac trunk. Next, the patient was positioned in the left lateral decubitus position, and a right thoracotomy with a muscle-sparing incision was made in the fourth intercostal space. The esophagus was resected after ligating and dissecting the azygos vein. Subsequently, the gastric tube was delivered into the thorax and a circular stapled end-to-side esophagogastric anastomosis was fashioned in the upper mediastinum. In addition, a nasogastric tube was positioned in the gastric tube to prevent vomiting and acute gastric tube distension.

Extended radical lymphadenectomy was performed during the right thoracic procedure including total mediastinal and upper abdominal lymphadenectomy. Total mediastinal lymph nodes, included the lymph nodes along the bilateral recurrent nerves (Video 1), and those typically resected during standard lymphadenectomy, including all lymph nodes in the middle and lower periesophageal portion, subcarinal region, and lower posterior mediastinum. The upper abdominal nodes included the paracardial, lesser curvature, greater curvature, left gastric, common hepatic, splenic, and celiac lymph nodes.

\section{Left Thoracic Procedure}

A single thoracic incision was made through the sixth or seventh intercostal space. The diaphragm was then incised to access and expose the abdominal cavity. The esophagus was mobilized, and the gastric tube (approximately $4 \mathrm{~cm}$ in width) was inserted along the greater curvature. The tumor was resected with at least $5 \mathrm{~cm}$ proximal clearance. Finally, an end-to-side esophagogastric anastomosis was fashioned with a circular staple at the subaortic or supra-aortic level. Subsequently, a feeding tube was inserted in the jejunum and a nasogastric tube was positioned in the gastric tube.

During the left thoracic procedure, limited lymphadenectomy was performed, removing the lymph nodes that were resected during standard lymphadenectomy. If accessible, lymph nodes in the upper mediastinum, adjacent to the upper esophagus or along the left recurrence nerve, were also removed in the surgical field. In this study, the left-sided thoracic approach, rather than the left thoracoabdominal incision, was used to reduce postoperative pain. However, the common hepatic and celiac nodes 
were not removed due to poor exposure and anatomical limitations through the left thoracic incision and due to the rarity of metastases in this region according to the map of lymph nodes metastases.

\section{Histologic Examination}

A frozen section procedure was not routinely performed during surgery. In this study, R0 resection was defined as complete tumor excision with all margins (circumferential, proximal, distal) $>1 \mathrm{~mm}$ free of tumor, based on postoperative histologic examination. Moreover, R1 resection involved microscopic residual tumors and $\mathrm{R} 2$ resection involved macroscopic residual tumors. All resected lymph nodes were labeled separately for pathologic examination according to the anatomical sites. All tumors were staged according to the TNM classification of the eighth edition of the American Joint Committee for Cancer staging manual.

\section{Follow-up and Outcomes}

Postoperative treatment was determined 1 month after surgery. Postoperative adjuvant chemotherapy (platinum-based) was recommended for patients with positive lymph nodes. The surgeon responsible for the operation decided whether postoperative radiotherapy was necessary based on resection margins.
Patients were seen in our outpatient clinic every 3 to 5 months during the first 2 years and every 6 months thereafter. If the patients did not return to our center, they were asked to undergo examinations at their local hospitals, and the follow-up data were obtained using telephone interviews with the patients or their family members. Oncologic follow-up consisted of CT of the chest and ultrasound of the upper abdomen and cervical region. Esophagoscopy was performed every year after surgery. Other examinations (eg, magnetic resonance imaging and bone scans) were performed according to clinical indications.

In this study, disease recurrence was defined as locoregional (including the anastomosis and surgical field) or distant (including distant organs and supraclavicular nodes) and was determined based on radiologic evidence and histologic sampling, if available. Data on patient outcomes were retrieved until August 2017 to ensure a minimum follow-up of 5 years.

The primary outcome for this analysis was overall survival (OS), calculated from the date of surgery when the allocation was revealed to the date of death from any cause. Disease-free survival (DFS) was defined as the period from the date of surgery to the date of first recurrence (locoregional, distant, or locoregional plus distant [mixed]) or death from any cause. Locoregional recurrence-free survival was defined as the period from the date of randomization to the date of first locoregional recurrence (locoregional only or mixed) or death from any cause. Metastasis-free

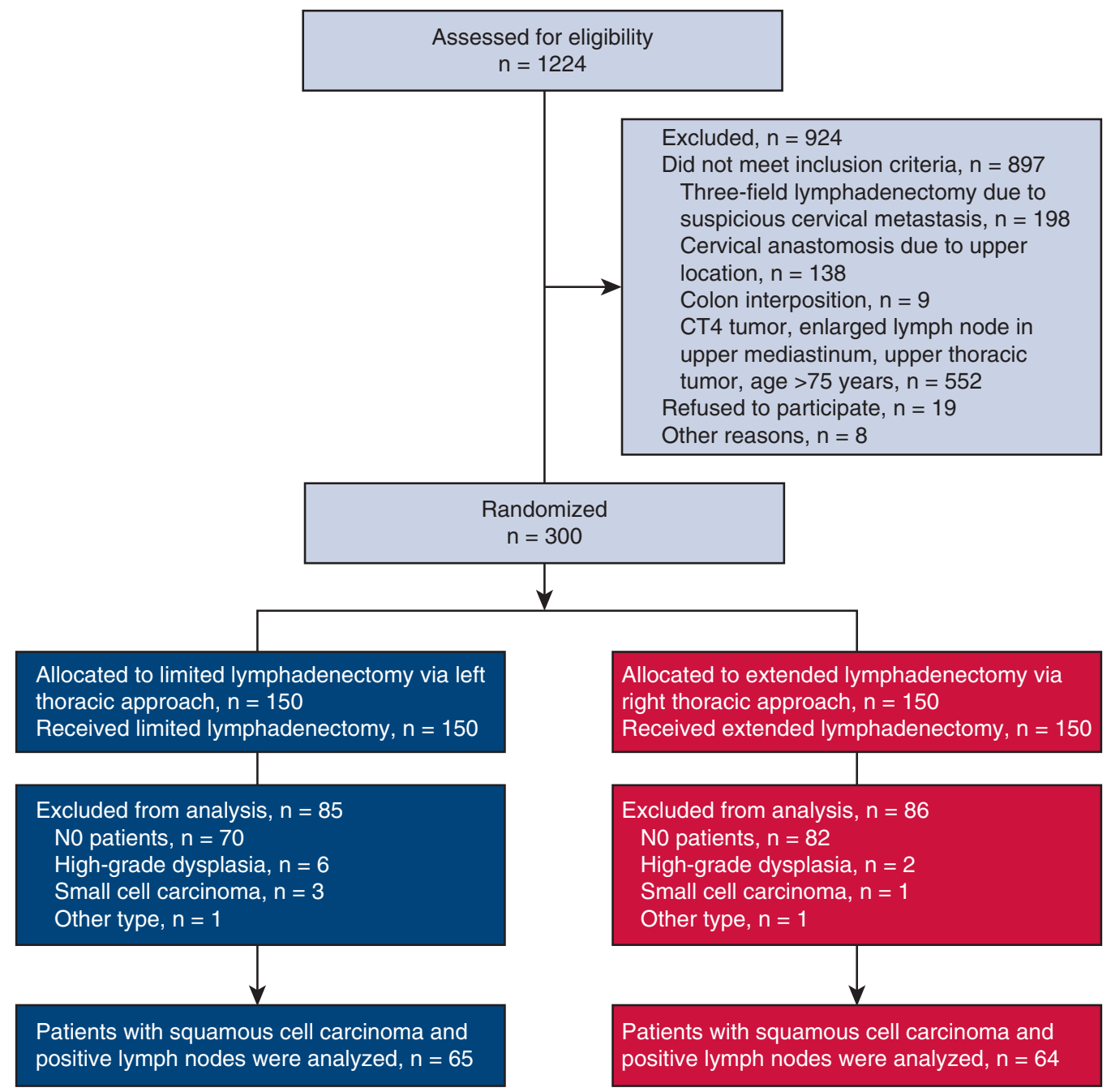

FIGURE 1. Flow diagram of the study. 
survival was calculated from the date of randomization to the date of first distant metastasis (distant only or mixed) or death from any cause.

\section{Statistical Analysis}

The sample size calculation of the original trial was previously described. ${ }^{6}$ Data were censored at the last follow-up. Survival curves were generated using the Kaplan-Meier method and were analyzed using the log-rank test. The median follow-up was calculated using reverse Kaplan-Meier estimates. Differences between the groups were calculated using the Mann-Whitney $U$ test, $\chi^{2}$ test, or Fisher exact test, as appropriate.

Since the 2 arms were not balanced in this post-hoc subgroup analysis, Cox regression was used to estimate the hazard ratios (HR) with $95 \%$ confidence interval (CI). Cox proportional hazards regression analyses using robust standard error technique were performed with the following variables: tumor location (middle vs lower), surgical approach (left vs right thoracic), resection margin (R0 vs R1-2), lymphovascular invasion (yes vs no), TNM stage (IIB-IIIB vs IV), and postoperative adjuvant treatment (none vs yes). We fit 2 multivariate Cox regression models to identify the risk factors. Model 1: only variables significantly associated with overall survival by univariate analysis were included. Model 2: all variables were included.

Analyses were performed using SPSS, version 17.0 (IBM Corp, Chicago, Ill) and Stata 15.0 software (StataCorp, College Station, Tex). Two-sided $P$-values $<.05$ were considered statistically significant.

\section{RESULTS}

\section{Patients' Characteristics}

Three hundred patients were enrolled in this study between May 2010 and July 2012. Of these, 150 were randomly assigned to the extended lymphadenectomy via right thoracic approach group and 150 to the limited

TABLE 1. Baseline demographic and clinical characteristics of the patients with esophageal squamous cell carcinoma

\begin{tabular}{|c|c|c|}
\hline & $\begin{array}{c}\text { Extended } \\
\text { lymphadenectomy } \\
(n=64)\end{array}$ & $\begin{array}{c}\text { Limited } \\
\text { lymphadenectomy } \\
(n=65)\end{array}$ \\
\hline $\begin{array}{l}\text { Age, } y, \text { median } \\
\quad \text { (interquartile range) }\end{array}$ & $60(54-66)$ & $59(55-63)$ \\
\hline \multicolumn{3}{|l|}{ Sex } \\
\hline Male & $51(79.7)$ & $53(81.5)$ \\
\hline Female & $13(20.3)$ & $12(18.5)$ \\
\hline \multicolumn{3}{|l|}{ BMI, $\mathrm{kg} / \mathrm{m}^{2}$} \\
\hline$<25$ & $50(78.1)$ & $46(70.8)$ \\
\hline$\geq 25$ & 14 (21.9) & $19(29.2)$ \\
\hline \multicolumn{3}{|l|}{ Comorbidities } \\
\hline Hypertension & $9(14.1)$ & $8(12.3)$ \\
\hline Diabetes mellitus & $1(1.6)$ & $6(9.2)$ \\
\hline Heart disease & $1(1.6)$ & $1(1.5)$ \\
\hline \multicolumn{3}{|l|}{ ASA score } \\
\hline $0 / 1$ & $45(70.3)$ & $51(78.5)$ \\
\hline $2 / 3$ & $19(29.7)$ & $14(21.5)$ \\
\hline \multicolumn{3}{|l|}{ Tumor location } \\
\hline Middle & $44(68.8)$ & $36(55.4)$ \\
\hline Lower & $20(31.3)$ & $29(44.6)$ \\
\hline
\end{tabular}

The data are presented as the number (percentage) unless otherwise specified. $B M I$, Body mass index; ASA, American Society of Anesthesiologists. lymphadenectomy via left thoracic approach group. Randomized patients with cancer other than squamous cell carcinoma (ie, high-grade dysplasia, small cell carcinoma, and carcinosarcoma) $(\mathrm{n}=14)$, and those without lymph nodes metastasis $(n=157)$, determined by postoperative pathology from the postoperative examination, were excluded from data analysis. Thus, the remaining 129 patients were included in the final data analysis, regardless of follow-up duration. Of these 129 patients, 64 patients were in the extended lymphadenectomy group and 65 in the limited lymphadenectomy group (Figure 1). As a post-hoc analysis, baseline characteristics were not balanced between the 2 groups with respect to body mass index, American Society of Anesthesiologist score, and tumor location (Table 1). Postoperative complications were comparable between the 2 groups, although the limited lymphadenectomy group had longer hospital stay than extended lymphadenectomy group (18, interquartile range [IQR], 15-23, vs 16, IQR, 14-19 days, $P<.001$, Mann-Whitney $U$ test). The total numbers of lymph nodes retrieved were 27 (IQR, 17-35) and 19 (IQR, 14-27) in the extended and limited lymphadenectomy groups, respectively $(P<.001$, Mann-Whitney $U$ test), whereas the TNM stages were comparable between the 2 groups. Fifteen patients $(23.4 \%)$ in the extended lymphadenectomy group had upper mediastinal LNM, whereas only $5(7.7 \%)$ in the limited lymphadenectomy group, $P=.013$ (Table 2), had upper mediastinal LNM. According to the study protocol, adjuvant treatment was recommended for patients with positive lymph nodes; however, $13(20.3 \%)$ and 16 $(24.6 \%)$ patients in the extended and limited lymphadenectomy group, respectively, did not undergo any adjuvant treatment due to poor physical status or financial reasons.

\section{DFS and OS}

The median follow-up for surviving patients was 68 months (range, 2-86 months; IQR, 62-82 months). As of the last follow-up, 39 of the 129 patients with lymph node metastasis, (extended lymphadenectomy group, $\mathrm{n}=22$; limited lymphadenectomy group, $\mathrm{n}=17$ ) were alive and disease-free (eventually without evidence of recurrence). The median DFS was 29 months in the extended lymphadenectomy group and 13 months in the limited lymphadenectomy group (HR, 1.542; 95\% CI, $1.018-2.335 ; P=.037$ ), with the cumulative 5-year DFS rates of $40 \%(95 \% \mathrm{CI}, 27 \%-52 \%)$ and $26 \%(95 \% \mathrm{CI}$, $15 \%-37 \%$ ), respectively (Figure $2, A$ ).

Furthermore, of the 129 patients with lymph node metastasis, 47 (extended lymphadenectomy group, $\mathrm{n}=27$; limited lymphadenectomy group, $\mathrm{n}=20$ ) were alive at the last follow-up. The median OS was 55 months in the extended lymphadenectomy group compared with 28 months in the limited lymphadenectomy group (HR, $1.719 ; 95 \%$ CI, 1.111-2.660; $P=.013$ ), with the 
TABLE 2. Surgical outcomes

\begin{tabular}{|c|c|c|c|}
\hline & $\begin{array}{l}\text { Extended lymphadenectomy } \\
\qquad(\mathrm{n}=64)\end{array}$ & $\begin{array}{l}\text { Limited lymphadenectomy } \\
\qquad(\mathrm{n}=65)\end{array}$ & $\boldsymbol{P}$ \\
\hline \multicolumn{4}{|l|}{ Postoperative complications } \\
\hline Anastomotic leakage & $1(1.6)$ & $3(4.6)$ & $.619 *$ \\
\hline Pulmonary infection & $9(13.1)$ & $10(15.4)$ & $.832 \dagger$ \\
\hline Cardiac complication & $9(13.1)$ & $8(12.3)$ & $.768+$ \\
\hline Chylothorax & $3(4.7)$ & $3(4.6)$ & $1.000^{*}$ \\
\hline Vocal cord paralysis & $2(3.1)$ & $4(6.2)$ & $.680 *$ \\
\hline In-hospital mortality & $0(0)$ & $2(3.1)$ & $.496 *$ \\
\hline Hospital stay, median (interquartile range) & $16(14-19)$ & $18(15-23)$ & $<.001 \ddagger$ \\
\hline Number of resected lymph nodes, median (interquartile range) & $27(17-35)$ & $19(14-27)$ & $<.001 \ddagger$ \\
\hline \multicolumn{4}{|l|}{ pT stage } \\
\hline $\mathrm{T} 1$ & $3(4.7)$ & $7(10.8)$ & .186 \\
\hline $\mathrm{T} 2$ & $27(42.2)$ & $18(27.7)$ & \\
\hline $\mathrm{T} 3$ & $32(50.0)$ & $35(53.8)$ & \\
\hline $\mathrm{T} 4$ & $2(3.1)$ & $5(7.7)$ & \\
\hline pN stage & & & $.561 \dagger$ \\
\hline N1 & $46(71.9)$ & $52(80.0)$ & \\
\hline $\mathrm{N} 2$ & $13(20.3)$ & $8(12.3)$ & \\
\hline $\mathrm{N} 3$ & $5(7.8)$ & $5(7.7)$ & \\
\hline \multicolumn{4}{|l|}{ Resection margin } \\
\hline R0 & $40(62.5)$ & $39(60.0)$ & $.771 \dagger$ \\
\hline $\mathrm{R} 1 / 2$ & $24(37.5)$ & $26(40.0)$ & \\
\hline Lymphovascular invasion & & & $.230 \dagger$ \\
\hline Yes & $23(35.9)$ & $17(26.2)$ & \\
\hline No & $41(64.1)$ & $48(73.8)$ & \\
\hline \multicolumn{4}{|l|}{ TNM stage } \\
\hline IIB & $2(3.1)$ & $6(9.2)$ & $.525 *$ \\
\hline IIIA & $20(31.3)$ & $17(26.2)$ & \\
\hline IIIB & $37(57.8)$ & $37(56.9)$ & \\
\hline IV & $5(7.8)$ & $5(7.7)$ & \\
\hline \multicolumn{4}{|l|}{ Region of lymph node metastasis } \\
\hline Upper mediastinum & $15(23.4)$ & $5(7.7)$ & $.013 \dagger$ \\
\hline Middle mediastinum & $22(34.4)$ & $17(26.2)$ & $.309+$ \\
\hline Lower mediastinum & $12(18.8)$ & $14(21.5)$ & $.693 \dagger$ \\
\hline Abdomen & $32(50.0)$ & $39(60.0)$ & $.254 \dagger$ \\
\hline \multicolumn{4}{|l|}{ Adjuvant therapy } \\
\hline None & $13(20.3)$ & $16(24.6)$ & $.593 *$ \\
\hline Chemotherapy & $38(59.4)$ & $31(47.7)$ & \\
\hline Radiotherapy & $3(4.7)$ & $5(7.7)$ & \\
\hline Chemoradiotherapy & $10(15.6)$ & $13(20.0)$ & \\
\hline
\end{tabular}

The data are presented as the number (percentage) unless otherwise specified. R0 was defined as a distance of $>1 \mathrm{~mm}$ from the radial (deep), proximal, or distal margins; $\mathrm{R} 1$ as microscopical residual tumors; and $\mathrm{R} 2$ as macroscopical residual tumors. $p T$, Pathologic tumor; $p N$, pathologic lymph node; $T N M$, tumor-node-metastasis. *Fisher exact test. $\dagger \chi^{2}$ test. $\ddagger$ Mann-Whitney $U$ test.

cumulative 5 -year OS rates of $51 \%(95 \%$ CI, 38\%-63\%) and $31 \%(95 \% \mathrm{CI}, 19 \%-42 \%)$, respectively (Figure $2, B)$.

Regression analysis of all included patients using a multivariate Cox proportional hazard model revealed independent associations of increased OS with extended lymphadenectomy, R0 resection margins, early TNM stage, and postoperative adjuvant therapy (Table 3 ).

DFS and OS for all the patients with esophageal squamous cell carcinoma in the trial, regardless of lymph nodes status, are available in Figure E1.

\section{Locoregional Recurrence-Free Survival and Distant Metastasis-Free Survival}

Recurrence type and DFS were further investigated. From randomization, 77 patients experienced recurrence. In patients from the extended lymphadenectomy group, 39 had recurrence, of whom 7 had locoregional progression, 27 had distant metastasis, 3 had mixed recurrence, and 2 had unclear recurrence. In patients from the limited lymphadenectomy group, 38 had recurrence, of whom 15 had locoregional recurrence, 15 had distant metastasis, 6 had 


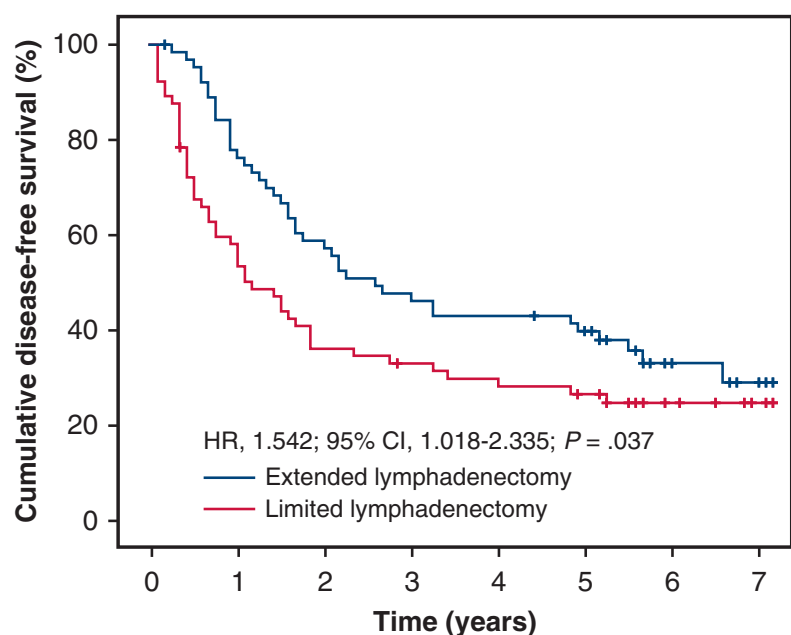

Numbers at risk

$\begin{array}{llllllll}\text { Extended } & 49 & 37 & 30 & 27 & 24 & 9 & 5 \\ & 37 & 23 & 20 & 18 & 15 & 7 & 3\end{array}$

95\% Confidence interval

Extended $\quad(66,87)(52,75) \quad(34,58)(31,55)(27,52)(21,45) \quad(16,42)$

Limited

$(46,70)(24,48)(21,45)(17,39)(15,37)(14,35)(14,35)$

A

FIGURE 2. Extended lymphadenectomy improved cumulative probability of disease-free survival (A) and overall survival (B) in patients with esophageal squamous cell carcinoma and lymph nodes metastasis. $H R$, Hazard ratio; $C I$, confidence interval.

mixed recurrence, and 2 had unclear recurrence. Extended lymphadenectomy significantly reduced locoregional recurrence (Table 4).

Patients in the extended lymphadenectomy group had significantly better locoregional recurrence-free survival compared with the limited lymphadenectomy group (HR, $0.583 ; 95 \%$ CI, 0.375-0.908; $P=.015$ ) (Figure 3, $A$ ). Metastasis-free survivals were not statistically significant (HR, 1.407; 95\% CI, 0.914-2.167; $P=.115$ ) but tended

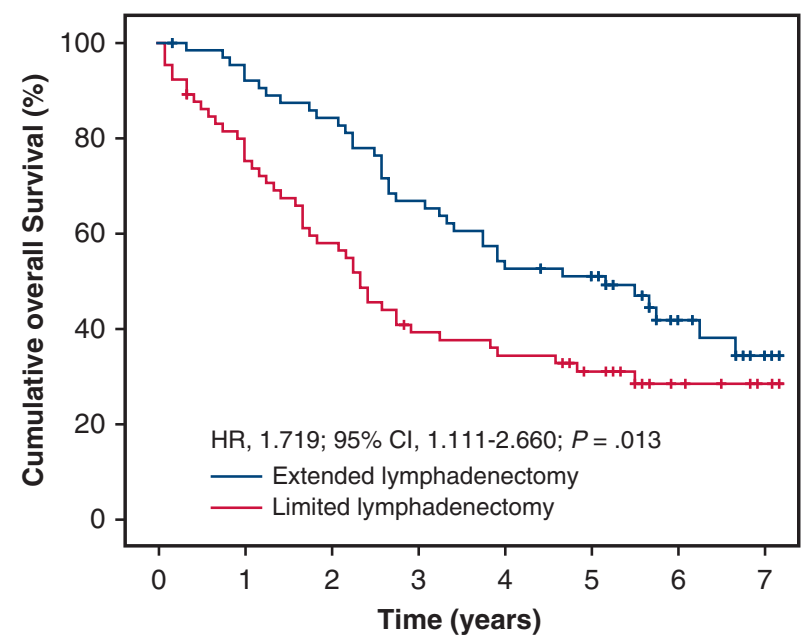

$\begin{array}{lccccccc}\begin{array}{l}\text { Numbers at risk } \\ \text { Extended }\end{array} & 60 & 53 & 42 & 34 & 31 & 13 & 6 \\ \text { Limited } & 51 & 37 & 24 & 21 & 16 & 7 & 3 \\ 95 \% \text { Confidence interval } & & & & & & \\ \text { Extended } & (85,99) & (75,93) & (55,78) & (40,65) & (38,63) & (28,55) & (20,48) \\ \text { Limited } & (70,90) & (46,70) & (27,51) & (22,46) & (19,42) & (17,40) & (17,40)\end{array}$

Limited $\quad(70,90)(46,70)(27,51)(22,46)(19,42)(17,40)(17,40)$

to be better in the extended lymphadenectomy group than the limited lymphadenectomy group (Figure 3,B).

\section{DISCUSSION}

In this long-term post-hoc survival report of esophageal squamous cell cancer patients with LNM, the extended lymphadenectomy via right thoracic approach had significantly better results than the limited lymphadenectomy via left thoracic approach after a minimum follow-up of

TABLE 3. Cox regression analyses of factors associated with overall survival

\begin{tabular}{|c|c|c|c|c|c|c|}
\hline & \multicolumn{2}{|c|}{ Univariate } & \multicolumn{2}{|c|}{ Multivariate (model 1*) } & \multicolumn{2}{|c|}{ Multivariate (model $2 \nmid$ ) } \\
\hline & $\overline{\text { HR }(95 \% \mathrm{CI})}$ & $P$ & HR $(95 \%$ CI $)$ & $P$ & HR $(95 \% \mathrm{CI})$ & $P$ \\
\hline Tumor location & 1.426 & & & & 1.232 & \\
\hline Lower/middle & $0.930-2.187$ & .104 & & & $0.788-1.927$ & .361 \\
\hline Extent of lymphadenectomy & 1.719 & & 1.893 & & 1.915 & \\
\hline Limited/extended & $1.118-2.642$ & .014 & $1.227-2.921$ & .004 & $1.211-3.029$ & .005 \\
\hline Resection margin & 1.692 & & 1.861 & & 2.103 & \\
\hline $\mathrm{R} 1-2 / \mathrm{R} 0$ & $1.099-2.605$ & .017 & $1.197-2.892$ & .006 & $1.310-3.377$ & .002 \\
\hline Lymphovascular invasion & 0.971 & & & & 0.896 & \\
\hline Yes/N0 & $0.617-1.527$ & .898 & & & $0.549-1.462$ & .660 \\
\hline TNM stage & 2.036 & & 2.341 & & 2.596 & \\
\hline IIB-IIIB/IV & $1.051-3.945$ & .035 & $1.256-4.362$ & .007 & $1.334-4.670$ & .001 \\
\hline Adjuvant therapy & 0.608 & & & & 0.001 & \\
\hline None/yes & $0.358-1.032$ & .065 & & & $1.443-4.670$ & .010 \\
\hline
\end{tabular}

R0 was defined as a distance of $>1 \mathrm{~mm}$ from the radial [deep], proximal, or distal margins; R1 as microscopical residual tumors; and R2 as macroscopical residual tumors. $H R$, Hazard ratio; $C I$, confidence interval. TNM, tumor-node-metastasis. * Model 1: variable with $P$ value $<.05$ by univariate analysis were included. $\dagger$ Model 2 : all variables were included. 
TABLE 4. Recurrence and metastasis sites in detail

\begin{tabular}{lccc}
\hline & $\begin{array}{c}\text { Extended } \\
\text { lymphadenectomy } \\
(\mathbf{n}=\mathbf{6 4})\end{array}$ & $\begin{array}{c}\text { Limited } \\
\text { lymphadenectomy } \\
(\mathbf{n}=\mathbf{6 5})\end{array}$ & $\boldsymbol{P}$ \\
\hline Recurrence types & & 15 & $.067 *$ \\
$\quad$ Locoregional & 7 & & \\
$\quad$ recurrence & & 15 & $.021^{*}$ \\
Distant metastasis & 27 & 6 & $.311^{*}$ \\
Mixed $\dagger$ & 3 & 2 & $1.000 \ddagger$ \\
Unclear & 2 & & \\
Recurrence sites & & 16 & $.129 *$ \\
Mediastinal & 9 & & \\
$\quad$ lymph nodes & & 2 & $1.000 \ddagger$ \\
Abdominal & 2 & & \\
$\quad$ lymph nodes & & 4 & $.744 \ddagger$ \\
Anastomotic site & 5 & 9 & $.451^{*}$ \\
Cervical lymph & 12 & & \\
$\quad$ nodes & & 3 & $.044 *$ \\
Lung & 10 & 3 & $.718 \ddagger$ \\
Liver & 4 & 6 & $.273 \ddagger$ \\
Bone & 2 & 0 & $.244 \ddagger$ \\
Ascites & 2 & 0 & $.496 \ddagger$ \\
Kidney & 1 & 0 & $.496 \ddagger$ \\
Skin & 1 & 0 & $.496 \ddagger$ \\
Thyroid & 1 & & \\
\hline
\end{tabular}

$* \chi^{2}$ test. $\dagger$ Patients with both locoregional recurrence and distant metastasis. $\ddagger$ Fisher exact test.

5 years, and the results of the present study (5-year survival) were consistent with our previous report (3-year survival)

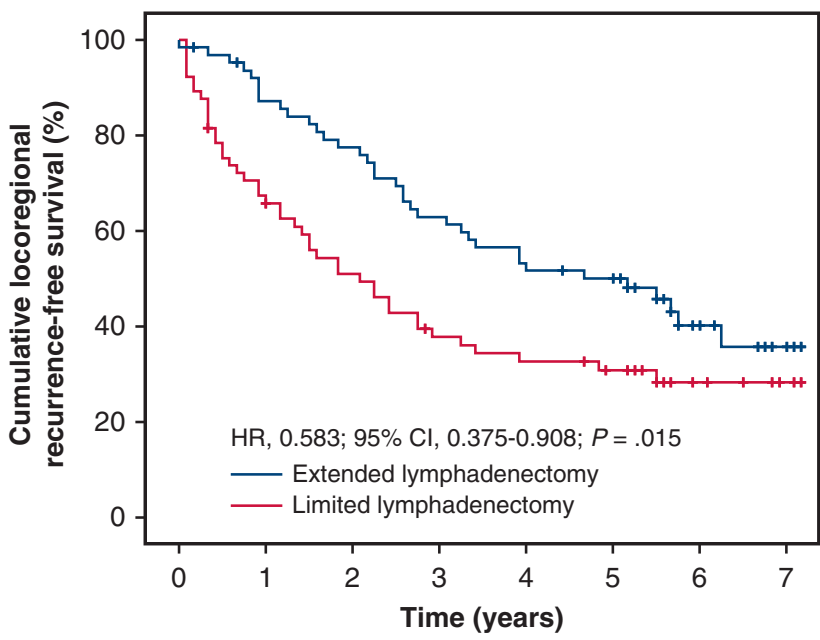

Numbers at risk

$\begin{array}{lccccccc}\text { Extended } & 54 & 48 & 39 & 33 & 30 & 11 & 5 \\ \text { Limited } & 42 & 31 & 22 & 19 & 16 & 7 & 3 \\ \text { 95\% Confidence interval } & & & & & & & \\ \text { Extended } & (79,95) & (67,88) & (51,75) & (39,64) & (37,63) & (36,61) & (21,50) \\ \text { Limited } & (54,77) & (39,63) & (26,50) & (21,44) & (19,42) & (17,40) & (17,40)\end{array}$

A and confirmed the advantage of radical extended lymphadenectomy for patients with advanced esophageal cancer.

China accounts for about one half of the cases of esophageal squamous cell carcinomas globally. ${ }^{8}$ In addition, more than one half of Chinese patients have lymphatic involvement at first diagnosis. Currently, transthoracic surgery combined with adjuvant treatment is still the main treatment strategy for esophageal cancer in China. The optimal surgical approach between the left and right thoracic approaches remains a subject of major debates. Most studies comparing the 2 approaches occurring in China used retrospective data. Ma and colleagues ${ }^{9}$ compared 167 Ivor-Lewis and 748 Sweet procedures, and the results showed comparable 5-year OS between the 2 approaches ( $48.4 \%$ vs $46.6 \%$, respectively; $P=.388)$. Liu and colleagues ${ }^{10}$ compared the Ivor-Lewis and Sweet surgical approaches in elderly patients using a propensity-matched method (57 pairs), and the results showed better OS $(54.6 \%$ vs $32.6 \%$, respectively; $P=.036)$ and DFS $(52.7 \%$ vs $20.2 \%$, respectively; $P=.021)$ associated with the right thoracic approach. The contradictory results between these studies were potentially due to patient selection bias and inadequate lymph nodes dissection during the Ivor-Lewis procedure. In our trial, we reported significantly improved 3-year OS of Ivor-Lewis procedure $(74 \%$ vs $60 \%, P=.029){ }^{6}$ Currently, after at a least follow-up of 5 years, the results of this study still support extended lymphadenectomy for

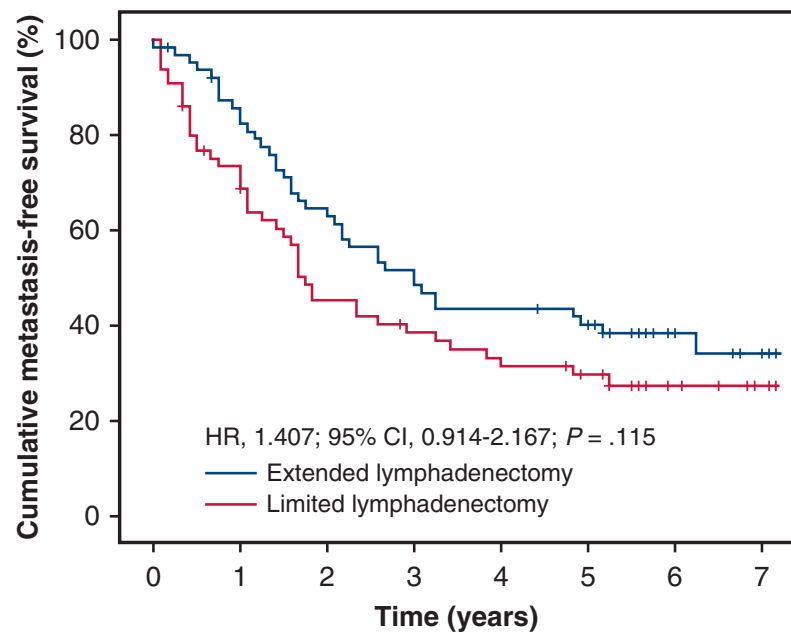

$\begin{array}{lccccccc}\begin{array}{l}\text { Numbers at risk } \\ \text { Extended }\end{array} & 53 & 40 & 32 & 27 & 24 & 10 & 1 \\ \text { Limited } & 45 & 27 & 22 & 19 & 15 & 7 & 3 \\ \text { 95\% Confidence interval } & & & & & & \\ \text { Extended } & (74,91) & (51,75) & (36,61) & (31,56) & (28,52) & (26,51) & (21,48) \\ \text { Limited } & (57,80) & (33,58) & (26,51) & (20,43) & (18,41) & (16,39) & (16,39)\end{array}$

B

FIGURE 3. Extended lymphadenectomy reduced cumulative probability of locoregional recurrence-free survival (A) and delayed metastasis-free survival (B) in patients with esophageal squamous cell carcinoma and lymph nodes metastasis. HR, Hazard ratio; $C I$, confidence interval. 
the treatment of esophageal squamous cell cancer, which was also verified by our previous results.

Locoregional recurrence and distant metastasis were the common reasons for the failure of treatment. In this study, extended radical lymph nodes dissection reduced locoregional recurrence, and delayed distant metastasis. Abdominal lymph nodes recurrence in this study were uncommon and comparable between the 2 arms, decreased mediastinal lymph node recurrence contributed to the reduced locoregional recurrence through radical upper mediastinal lymphadenectomy. Although distant metastasis reduction was nonsignificant, the cumulative distant metastasis-free survival trended to be better after extended radical lymph nodes dissection throughout the follow-up period, indicating the necessity of lymphadenectomy for advanced esophageal squamous cell carcinoma.

Tumor stage is associated with recurrence. ${ }^{11}$ Recent studies showed that preoperative chemo/radiotherapy could downstage the esophageal cancer, and neoadjuvant treatment was established as the standard of care for locally advanced esophageal cancer. In the Chemoradiotherapy for Oesophageal Cancer followed by Surgery Study (CROSS), patients in the neoadjuvant chemoradiotherapy plus surgery group had lower incidence of lymph node metastasis (31 vs $75 \%$, respectively; $P<.001)$ compared with surgery alone. ${ }^{12}$ Long-term follow-up in the CROSS trial showed that both locoregional and distant disease control significantly improved in the neoadjuvant chemoradiotherapy plus surgery group. ${ }^{13,14}$ However, 2 issues should be noted. First, in previous trials from Western countries, transthoracic procedure, and transhiatal with or without mediastinal lymphadenectomy, were both applied, and the majority of patients had adenocarcinoma. ${ }^{12,15,16}$ Second, regardless of lymph node status, survival after neoadjuvant therapy in previous trials ranged from $23 \%$ to $47 \%, 8,13,14$ whereas in this study, the 5 -year OS of esophageal squamous cell carcinoma with LNM after extended lymphadenectomy was $51 \%$. Many factors may explain the different results, including differences in surgical techniques and tumor biology. However, radical lymphadenectomy via thoracic approach can be an important factor contributing to improved survival.

Resection margin was another factor associated with patient survival. Patients with R0 resection, including negative proximal, distal, and circumferential resection margins, had better survival. In this study, the Royal College of Pathologists criteria were used to define a positive circumferential resection margin when a tumor is found $<1 \mathrm{~mm}$ of the lateral margin, consistent with previous report ranging from $20.3 \%$ to $67.4 \% .{ }^{17}$ Although adjuvant treatment was identified as a risk factor related to improved survival, it should be noted that this trial was not designed to investigate adjuvant treatment, and that patients who had poor physical status and those with postoperative complications did not undergo adjuvant treatment. Neoadjuvant treatment significantly increased R0 resections after surgery. ${ }^{13}$ Compared with postoperative adjuvant treatment, neoadjuvant treatment improved patient survival. ${ }^{18}$ Thus, future trials are needed to clarify the role of extended radical lymphadenectomy combined with neoadjuvant treatment for patients with esophageal squamous cell carcinoma.

This study was limited by the patients included in both groups, which may have been underpowered for a robust analysis, as the study was primarily powered on 3-year survivals of clinically resectable thoracic esophageal cancer, rather than only cancers with LNM. However, tumor location, the depth of tumor invasion, and resection margin showed no differences between the 2 groups, supporting the validity of this analysis. The study results, which were based on postoperative pathologic examination, were also limitations. The accurate detection of LNM is necessary for determining the proper treatment strategy. We previous reported that LNM was associated with tumor length, tumor invasion depth, tumor differentiation, and lymphovascular invasion. ${ }^{19}$ However, detection of accurate LNM status is difficult due to the limitations of current imaging modalities. ${ }^{20}$ In this study, preoperative oncologic evaluation was assessed based on thoracic and abdominal enhanced CT and cervical and abdominal ultrasound. In a meta-analysis comparing the diagnostic tools for esophageal cancer, ${ }^{21}$ endoscopic ultrasound was the most sensitive tool for detecting regional lymph node metastasis, whereas CT and positron emission tomography were more specific tests. Therefore, extended radical lymphadenectomy would be the preferred option for patients with suspicious lymphatic involvement as determined by accurate preoperative staging by the combined use of endoscopic ultrasound and positron emission tomography. Moreover, frozen section of lymph nodes during surgery can be another option, and extended radical lymph nodes dissection may be indicated when positive lymph nodes are proven by frozen section. For patients without suspicious lymphatic involvement as assessed by careful preoperative examinations and frozen section, extended dissection may not be necessary.

In conclusion, after a minimum of 5-year follow-up, the results of this study verified better survival after extended lymphadenectomy for patients with esophageal squamous cell carcinoma and lymphatic involvement. Compared with limited lymphadenectomy, less locoregional recurrences occurred after extended radical lymphadenectomy.

\section{Conflict of Interest Statement}

Authors have nothing to disclose with regard to commercial support.

\section{References}

1. Omloo JM, Lagarde SM, Hulscher JB, Reitsma JB, Fockens P, van Dekken H, et al. Extended transthoracic resection compared with limited transhiatal 
resection for adenocarcinoma of the mid/distal esophagus: five-year survival of a randomized clinical trial. Ann Surg. 2007;246:992-1000.

2. Sjoquist KM, Burmeister BH, Smithers BM, Zalcberg JR, Simes RJ, Barbour A, et al. Survival after neoadjuvant chemotherapy or chemoradiotherapy for resectable oesophageal carcinoma: an updated meta-analysis. Lancet Oncol. 2011;12:681-92.

3. Hulscher JB, van Sandick JW, de Boer AG, Wijnhoven BP, Tijssen JG, Fockens P, et al. Extended transthoracic resection compared with limited transhiatal resection for adenocarcinoma of the esophagus. N Engl J Med. 2002;347:1662-9.

4. Malhotra GK, Yanala U, Ravipati A, Follet M, Vijayakumar M, Are C. Global trends in esophageal cancer. J Surg Oncol. 2017;115:564-79.

5. Li B, Xiang J, Zhang Y, Li H, Zhang J, Sun Y, et al. Comparison of Ivor-Lewis vs Sweet esophagectomy for esophageal squamous cell carcinoma: a randomized clinical trial. JAMA Surg. 2015;150:292-8.

6. Li B, Hu H, Zhang Y, Zhang J, Miao L, Ma L, et al. Extended right thoracic approach compared with limited left thoracic approach for patients with middle and lower esophageal squamous cell carcinoma: three-year survival of a prospective, randomized, open-label trial. Ann Surg. 2018;267:826-32.

7. Rice TW, Ishwaran H, Ferguson MK, Blackstone EH, Goldstraw P. Cancer of the esophagus and esophagogastric Junction: an eighth edition staging primer. J Thorac Oncol. 2017;12:36-42.

8. Di Pardo BJ, Bronson NW, Diggs BS, Thomas CR Jr, Hunter JG, Dolan JP. The global burden of esophageal cancer: a disability-adjusted life-year approach. World J Surg. 2016;40:395-401.

9. Ma J, Zhan C, Wang L, Jiang W, Zhang Y, Shi Y, et al. The sweet approach is still worthwhile in modern esophagectomy. Ann Thorac Surg. 2014;97:1728-33.

10. Liu Q, Chen J, Wen J, Yang H, Hu Y, Luo K, et al. Comparison of right- and left-approach esophagectomy for elderly patients with operable thoracic esophageal squamous cell carcinoma: a propensity matched study. J Thorac Dis. 2017;9:1883-90

11. Lee PC, Mirza FM, Port JL, Stiles BM, Paul S, Christos P, et al. Predictors of recurrence and disease-free survival in patients with completely resected esophageal carcinoma. J Thorac Cardiovasc Surg. 2011;141:1196-206.

12. van Hagen $\mathrm{P}$, Hulshof $\mathrm{MC}$, van Lanschot JJ, teyerberg EW, van Berge Henegouwen MI, Wijnhoven BP, et al. Preoperative chemoradiotherapy for esophageal or junctional cancer. N Engl J Med. 2012;366:2074-84.

13. Shapiro J, van Lanschot JJB, Hulshof M, van Hagen P, van Berge Henegouwen MI, Wijnhoven BPL, et al. Neoadjuvant chemoradiotherapy plus surgery versus surgery alone for oesophageal or junctional cancer (CROSS): long-term results of a randomised controlled trial. Lancet Oncol. 2015;16:1090-8.

14. Oppedijk V, van der Gaast A, van Lanschot JJ, van Hagen P, van Os R, van Rij CM, et al. Patterns of recurrence after surgery alone versus preoperative chemoradiotherapy and surgery in the CROSS trials. J Clin Oncol. 2014;32: 385-91.

15. Orringer MB, Marshall B, Chang AC, Lee J, Pickens A, Lau CL. Two thousand transhiatal esophagectomies: changing trends, lessons learned. Ann Surg. 2007; 246:363-72; discussion 72-4.

16. Kelsen DP, Winter KA, Gunderson LL, Mortimer J, Estes NC, Haller DG et al. Long-term results of RTOG trial 8911 (USA Intergroup 113): a random assignment trial comparison of chemotherapy followed by surgery compared with surgery alone for esophageal cancer. J Clin Oncol. 2007 25:3719-25.

17. Wu J, Chen QX, Teng LS, Krasna MJ. Prognostic significance of positive circumferential resection margin in esophageal cancer: a systematic review and meta-analysis. Ann Thorac Surg. 2014;97:446-53.

18. Ando N, Kato H, Igaki H, Shinoda M, Ozawa S, Shimizu H, et al. A randomized trial comparing postoperative adjuvant chemotherapy with cisplatin anc 5-fluorouracil versus preoperative chemotherapy for localized advanced squamous cell carcinoma of the thoracic esophagus (JCOG9907). Ann Surg Oncol. 2012;19:68-74.

19. Li B, Chen H, Xiang J, Zhang Y, Li C, Hu H, et al. Pattern of lymphatic spread in thoracic esophageal squamous cell carcinoma: a single-institution experience. J Thorac Cardiovasc Surg. 2012;144:778-85.

20. Sgourakis G, Gockel I, Lyros O, Hansen T, Mildenberger P, Lang H. Detection of lymph node metastases in esophageal cancer. Expert Rev Anticancer Ther. 2011; 11:601-12.

21. van Vliet EP, Heijenbrok-Kal MH, Hunink MG, Kuipers EJ, Siersema PD Staging investigations for oesophageal cancer: a meta-analysis. $\mathrm{Br} J$ Cancer. 2008;98:547-57.

Key Words: esophageal cancer, squamous cell carcinoma, esophagectomy, lymphadenectomy, lympha nodes metastasis, post-hoc analysis, survival 


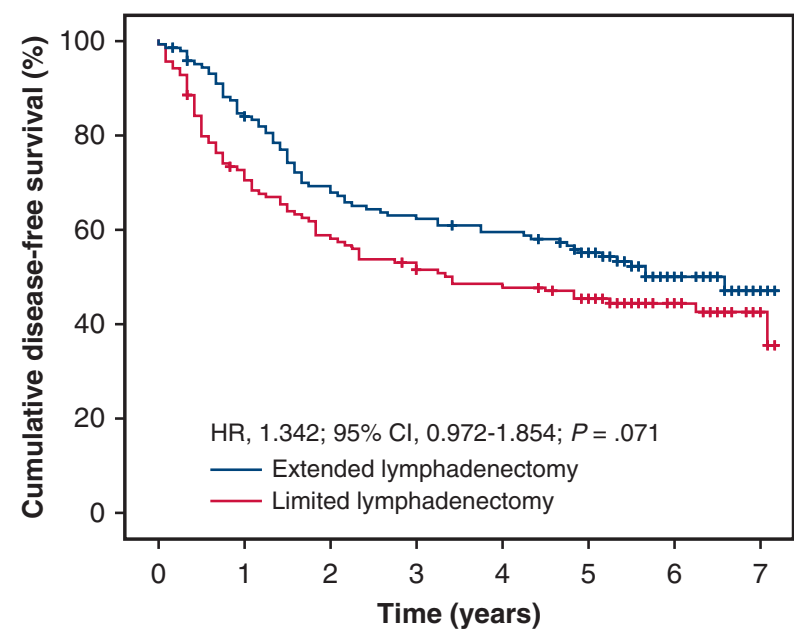

Numbers at risk

$\begin{array}{llllllll}\text { Extended } & 123 & 99 & 90 & 84 & 72 & 33 & 7 \\ \text { Limited } & 100 & 81 & 71 & 64 & 56 & 27 & 7\end{array}$

95\% Confidence interval

Extended $\quad(78,90)(62,77)(54,70)(51,68)(47,63)(41,59) \quad(37,57)$

A

Limited $\quad(65,80)(50,66)(43,60)(39,56)(37,54)(36,53)(34,51)$

FIGURE E1. Extended lymphadenectomy nonsignificantly improved cumulative probability of DFS (A) and OS (B) in all the 286 patients with esophageal
squamous cell carcinoma in the trial after a minimum follow-up of 5 years. The median DFS was 79 months in the extended lymphadenectomy group compared with 41 months in the limited lymphadenectomy group, and the cumulative 5-year DFS rates for extended and limited lymphadenectomy group were 55\% (95\% CI, 47\%-63\%) and 45\% (95\% CI, 37\%-54\%), respectively. The median OS was unreached in the extended lymphadenectomy group compared with 85 months in the limited lymphadenectomy group, and the cumulative 5-year OS rates were 63\% (95\% CI, 56\%-71\%), and 53\% (95\% CI, 44\%-61\%), respectively. HR, Hazard ratio; $C I$, confidence interval. 